\title{
Pesquizas helminthologicas realisadas em 5amburg
III. Trematodeos dos generos Pneumonoeces e Ostiolum
}

\author{
por \\ LAURO TRAVASSOS E ANTONIO R. DARRIBA
}

(Com as estampas LXVI-LXXI).

Os trematodeos do pulmão dos batrachios são constituidos por dois grupos: o primeiro pelo genero Pneumonoeces, mais tarde desdobrado e o segundo pelo genero Haplometra.

Vamos nos occupar do primeiro grupo nomeado.

O genero Pneumonoeces foi estabelecido por Looss em 1899, que o denominou de Haematoleuchus, nome occupado e por isso mudado em 1902, para Pneumonoeces. Quando Looss estabeleceu o genero incluio nelle apenas 3 especies denominadas $P$. variegatus, $P$. similis $e P$. asper. Estas tres especies tinham sido antes (1894) estudadas como sendo apenas uma, o que acarretou algumas confusões de auctores que posteriormente se occuparam com estes parasitos; assim Luehe reproduz com o nome de $P$. variegatus uma figura de Looss que este auctor diz corresponder ao similis, mas que nos parece dever corresponder talvez ao asper. Das especies europeas não mais se fez um estudo de revisão. Wundsch em 1911 em sua these de doutoramento caracterisou bem o asper e descreve, aliás de um modo menos recommendavel, pois limitou-se a fazer a diagnose differencial, uma nova especie a que denominou Pneumonoeces schulzei.

Deste modo as especies deste genero encontradas na Europa ficam sendo 4. $\mathrm{Na}$ America do Norte, Stafford descrevey as seguintes: $P$. breviplexus, $P$. variople$x u s, P$. s.miliplexus, $P$. longiplexus, e $P$.medioplexus. Varioplexus e similiplexus são consideradas identicas. As duas ultimas citadas foram separadas de Pneumonoeces longiplexus para constituir o typo do genero Pneumobites Ward, 1917 e medioplexus por ser identica a Ostiolum formosum, typo do genero Ostiolum Pratt, 1903.

O genero de Ward a nosso vêr não póde ser mantido.

Em 1905, Klein descreve uma especie da India, $P$. campyristis.

Em 1906 Seely descreve com o nome de $P$. complexus uma outra especie norte americana. Johnston (1912), descreve uma da Australia com o nome de $P$. australis. Cort, em 1917, descreve com o nome de $P$. coloradensis mais uma especie que deve ser incluida no genero Ostiolum. Travassos e Artigas descrevem em 1927 a primeira especie sul americana $P$. neivai. Lutz, em 1928 denominou de $P$. pla- 
norbinus o parasito commum no pulmão das rãs do Rio de Janeiro e no mesmo trabalho dá uma figura de $P$. neivai, referida na explicação das figuras com o nome de $P$. pseudis sem qualquer outra referencia no texto. Finalmente Irwin em 1929, descreve mais uma nova especie norte americana $P$. parviplexus. Resumindo verificámos ser 17 o numero de especies mencionadas na bibliographia para o grupo Preumonoeces e mais uma que descrevemos agora. Destas, algumas nos parecem synonymas, como veremos adiante.

São as seguintes as especies incluidas no genero Pneumonoeces:

variegatus (Zeder, 1800)

similis (Looss, 1899)

asper (Looss, 1899)

longiplexus (Stafford, 1902) (Pneumobites)

breviplexus (Stafford, 1902)

varioplexus (Stafford, 1902) (=similiplexus)

similiplexus (Stafford, 1902) (=varioplexus)

medioplexus (Stafford, 1902) (=Ostiolum formosum)

campyristis Klein, 1905

complexus Seely, 1906 (Ostiolum)

schulzei Wundsch, 1911

australis Johnston, 1912

coloradensis Cort, 1917 (Ostiolum)

neivai Travassos \& Artigas, 1927

planorbinus Lutz, 1929 (n. n.) (=neivai) pseudis Lutz, 1929 (só figura) (=neivai) parviplexus Irwin, 1929

fuelleborni $\mathbf{n}$. sp.

Fazendo um estudo comparativo cuidadoso entre estas diversas especies notamos logo dois typos fundamentaes-um caracterisado pela presença de duas alças uterinas longitudinaes, situadas na area extra-cecal e outro no qual estas alças extracecaes não existem. Esta conformação do utero é muito curiosa e a eschematisamos nas figuras 1 e 2. Estes dois ty. pos uterinos caracterisam os generos Pneumonoeces e Ostiolum.

No grupo onde existe a alça extracecal a porção ascendente e descendente do utero formam alças transversaes muito largas e que geralmente ultrapassam os campos cecaes nos dois lados. Neste grupo observam-se variações enormes nas dimensões das alças longitudinaes e, baseando-se nisto, Ward propoz o genero Pneumobites, que como dissemos não deve ser mantido. Observamos os dois typos extremos de desenvolvimento da alça longitudinal nas duas especies: longiplexus e fuelleborni. Esta ultima especie pode ser interpretada a primeira vista como uma fórma intermediaria entre Preumonoeces e Ostiolum. $\mathrm{O}$ exame mais attencioso, mostra, pelo aspecto geral do ulero, que apenas houve uma reduccão nas dimensões das alças sem haver a disposição que caracterisa Ostiolum.

Um outro orgão que apresenta typos estructuraes diversos são os vitellinos. Nos diversos Pneumonoeces observam-se dois typos de vitellinos-um observado em variegatus e no qual os folliculos são dispostos por grupos em fórma de "roseta" e se estendem de um modo mais ou menos symetrico na porção anterior e posierior do corpo, e outro typo em que que a disposição em roseta não é evidente, ficando os folliculos apenas na metade anterior do corpo, é o typo observado em similis. Pelo criterio de Witenberg este caracter seria sufficiente para um novo genero o que não parece razoavel.

Entre as diversas especies de Pneumonoeces destacam-se o $P$. campyristis pela reduzida bolsa do cirro, que em todas as outras é extremamente a longada. O acetabulo é tambem susceptivel de grandes variações, aliás é sempre mediocre; o maior tamanho relativo é encontrado em schulzei e em neivai falta completamente. $\mathrm{O}$ revestimento cuticular póde ser guarnecido de espinhos em 
pequeno numero; em variegafus, breviplexus, campyristis, australis, neivai e fuelleborni faltam.

No genero Ostiolum tambem os espinhos faltam em uma especie-complexus.

A posição dos testiculos, invocada por Ward como um dos elementos genericos de Preunobiles, não nos parece razoavel pois podemos observar todas as modalidades, sendo que neivai póde apresentar os dois typos dependendo do gráo da contractura. Aliás neivai se approxima muito, pelas alças longitudinaes do utero, de $P$. longiplexus. Um factor importante para a caracterisação dos Pneumonoeces é o tamanho dos ovos. Foi este o caracter fundamental de Looss para separar as tres primeiras especies européas e é ainda este caracter que permitte bem separar asper de schulzei. De um modo geral os Pneumonoeces se pódem bem caracterisar pelos seguintes elementos: comprimento relativo da porção anterior do corpo (pré-acetabular), disposi. ção dos vitellinos, comprimento relativo das alças longitudinaes do utero, relação entre as ventosas, dimensões dos ovos e pelos espinhos cuticulares.

A evolução ainda não foi descripta de um modo claro para nenhuma especie.

Pneumonoeces Looss, 1902.

Distomum auctor. p. p.

Haematoleuchus Looss, 1899, p. 600.

Haematoleuchus Luehe, 1900, p. 557 e 561.

Haematoleuchus Stiles, 1901, p. 189.

Haemaioleuchus Stiles \& Hassal, 1902, p. 20.

Haematoleuchus Stafford, 1902, p. 895.

Haematoleuchus Odhner, 1902, p. 45.

Haematoleuchus Pratt, 1902, p. 888.

Haematoleuchus Stafford, 1902, p. 418.
Haematoleuchus Stafford, 1902, p. 725.

Preumònoeces Looss, 1902, p. 732. Pneumonoeces Pratt, 1903, p. 37.

Preumonoeces Klein, 1905, p. 64.

Haematoleuchus Klein, 1905, p. 64.

Pneumonoeces Stafford, 1905, p. 687.

Pneumonoeces Luehe, 1909, p. 90 e 102.

Pneumonoeces Wundsch, 1911, p. 5. Pneumonoeces Cort, 1915, p. 212.

Pneumóbites Ward, 1917, p. 5.

Pneumobiles Ward, 1918, p. 403.

Pneumonoeces Ward, 1918, p. 403.

Pneumonoeces Travassos \& Artigas, 1927, p. 214 (erro).

Pneumonoeces Fuhrmann, 1928, p. 112.

Diagnose: Plagiorchidae: Saphedrinae.-Trematodeos de tamanho médio, com cuticula lisa ou revestida de espinhos; acetabulo reduzido ou ausente; póro genital na zona do pharynge; bolsa do cirro muito longa com cirro, prostata e longa vesicula seminal; testiculos intracecaes, ellipsoides mais ou menos allongados, com zonas e campos geralmente coincidindo parcialmente; utero com o ramo descendente formando na extremidade posterior duas alças longitudinaes extra-cecaes mais ou menos longas; ovos de casca escura quasi negra; vitellinos com folliculos de dimensões médias, geralmente formando rosetas, dorsaes, extra e intra-cecaes.

Habitat: pulmão de anuros.

Especie typo: $P$. variegatus (Rudolphi, 1819).

Pneumonoeces variegatus (Rudolphi, 1819).

(Est. LXVI, figs. 3-4)

Distoma variegatum Rudolphi, 1819, p. 99 e $378, p . p$.

Distoma (Brachylaimus) variegatus Dujardin, 1845 , p. 416 , p. p.

Distoma variegatum Pagent, 1857, p. 41, pl. 5, fig. $2, p . p$. 
Distoma variegatum Molin, 1859, p. 828 , pl. 3, fig. 2 , p. p.

Distoma variegatus Looss, 1892, p. $65, p . p$.

Distoma variegatus Looss, 1893, p. 812 , etc. fig. $3, p . p$.

Distoma cylindraceum Pachinger, 1888 , p. 105 , etc. fig. B. C. pl. I, p. p. (segundo Looss).

Disfomum variegatum Looss, 1894, p. 71 , etc. pl. II, fig. $45,46,47$ ?, 48 , pl. VII, fig. 135 , (pl. VII, fig 135, (pl. VII, figs. $134,136-146$ ?); p. $p$.

Distoma variegatum Luehe, 1899, p. $533, p$. $p$.

Haematoleuchus variegatus Looss, 1899, p. 601.

Haematoleuchus variegatus Luehe, 1900 , p. 556.

Haematoleuchus variegatus Stiles, 1901, p. 178.

Disioma variegatum Stiles \& Hassal, 1902, p. 20.

Dis to ma variegatum Gronkowski, 1902 , p. 511, 518, 531, pl. 13, figs. $3,11,12$.

Pneumonoeces variegatus Looss, 1902, p. 806.

Haematoleuchus variegatus Looss, 1902 , p. 429.

Distomum variegatum Stafford, 1902, p. 895 .

Haematoleuchus variegatus Stafford, 1902 , p. 896.

Preumonoeces variegatus Klein, 1905, p. 59 , etc.

Haema'oleuchus variegatus Ssinitzin, 1905, p. 137.

Haematoleuchus variegatus Ssinitzin, 1906, p. 686.

Distomum variegatum Ssinitzin, 1907, p. 34.

Preumonoeces variegat us Luehe, 1909, p. 104 (não a figura 82).

Pneumonoeces variegatus Wundsch, 1911, p. 40.
Pneumonoeces variegatus Johnston, 1912, p. 286.

Distomum variegatum Cort, 1915, p. p. 203.

Distomum variegatus Nicoll, 1926, p. 15.

Pneumonoeces variegatus Fuhrmann, 1928 , p. 68 , fig. 89 .

Esta especie é a mais caracteristica por apresentar a porção do corpo préacetabular muito alongada. E' a maior especie do genero. Corpo alongado, claviforme, sendo a porção pré-acetabular muito mais delgada que a posterior. A cuticula é lisa e o acetabulo bem desenvolvido e mais ou menos equatorial ou logo adiante do meio do corpo, é menor que a ventosa oral. Ventosa oral sub-terminal, seguida logo pelo pharynge que é forte. Esophago curto e delgado. Cecos estendendo-se até perto da extremidade posterior, são largos e pouco sinuosos. Póro genital na zona pharyngeana. Bolsa do cirro delgada e extremamente longa, sem comtudo attingir o acetabulo; contêm cirro, prostata e longa e sinuosa vesicula seminal. Os testiculos são ellipsoides alongados, post-equatoriaes, com zonas e campos coincidindo mais ou menos, conforme o estado de contractura do corpo. Ovario igualmente ellipsoide alongado, podendo apresentar algumas vezes lobulações pouco profundas; anteriormente attinge a zona acetabular e posteriormente a zona do testiculo anterior; é situado lateralmente. Espermatheca muito desenvolvida, tão grande como o ovario, é situada medianamente na zona ovariana e com area coincidindo parcialmente. Glandula de Mehlis muito desenvolvida. Utero constituido por um ramo descendente que fórma alças transversaes mais ou menos amplas até attingir a extremidade posterior do corpo, mantendo-se sempre na area intra cecal; após attingir a extremidade posterior fórma duas grandes alças longitudinaes extra- 
cecaes, a direita e a esquerda do corpo, estas alças são muito desenvolvidas e attingem a zona ovariana, isto é, mais ou menos o equador do corpo. Após a formação das alças o utero se dirige para deante, pela area intra-cecal, formando sinuosidades e pequenas alças para terminar, por uma vagina pouco desenvolvida, no póro genital. Os ovos contidos no utero são de côr castanho escura tornando-se quasi negros na porção terminal; são operculados e apresentam ainda no utero embrião formado. Os vitellinos são dispostos por grupos de folliculos formando rosetas dispostas dorsalmente na area cecal, intra e extra-cecal, desde logo abaixo da bifurcação do apparelho digesitivo até perto da terminação dos cecos, em regra ultrapassando a zona testicular. Dos diversos exemplares medidos organisamos o seguinte quadro de dimensões:

\begin{tabular}{l|r|r|r|r|r|r}
\hline \hline Comprimento & $8,56 \mathrm{~mm}$ & 7,18 & 7,75 & 7,06 & 8,50 & 11,50 \\
Largura & 1,37 & 1,25 & 1,43 & 1,31 & 1,37 & 2,18 \\
Acetabulo & $0,52 \times 0,44$ & $0,27 \times 0,22$ & $0,28 \times 0,28$ & $0,19 \times 0,17$ & $0,31 \times 0,31$ & $0,34 \times 0,34$ \\
Ventosa oral & $0,47 \times 0,44$ & $0,42 \times 0,41$ & $0,41 \times 0,42$ & $0,40 \times 0,40$ & $0,45 \times 0,42$ & $0,47 \times 0,47$ \\
Pharynge & $0,21 \times 0,22$ & $0,17 \times 0,17$ & $0,17 \times 0,21$ & $0,18 \times 0,20$ & $0,21 \times 0,21$ & $0,21 \times 0,21$ \\
Testiculos & $1, \times 0,57$ & $0,92 \times 0,45$ & $1,1 \times 0,42$ & $0,8 \times 0,5$ & $1, \times 0,6$ & $1,1 \times 0,4$ \\
Ovario & $1,2 \times 0,50$ & $1, \times 0,50$ & $1,1 \times 0,55$ & $1, \times 0,5$ & $1,1 \times 0,6$ & $1,5 \times 0,4$ \\
Ovos & $0,61 \times 0,37$ & $0,74 \times 0,28$ & $0,80 \times 0,35$ & $0,71 \times 0,24$ & $0,85 \times 0,35$ & $0,95 \times 0,35$ \\
& $0,024-0,028$ & $0,028 \times$ & $0,024 \times$ & $0,028 \times$ & $0,024-0,026$ & 0,028 a 0,032 \\
\hline \hline
\end{tabular}

Habitat: pulmão de Rana temporaria L., R. esculenta L. e Colopteryx virgo.

\section{Destribuição geographica: Europa.}

Examinámos desta especie material proveniente de Hamburgo, por nós colleccionado, que nos foi dado pelo Dr. Hoeppli e que existia no Museu Zoologico de Hamburgo. Examinámos tambem material de Rana esculenta ridibunda Pall., colleccionado no Turquestão Russo pelo Dr. M. Strom. Entre os exemplares examinados tivemos a opportunidade de observar um (fig. 4) que apresentava anomalia das glandulas genitaes representada por um desdobramento dos testiculos e do ovario. O testiculo anterior tinha a porção separada em um pequeno corpo redondo e o testiculo posterior era duplo; media o testiculo anterior 1,14 $\times 0,42 \mathrm{~mm}$. e corpuscula anterior $0,28 \times$ $0,25 \mathrm{~mm}$., os testiculos posteriores me- diam respectivamente $1,17 \times 0,55$ e 0,51 $\times 0,35 \mathrm{~mm}$. O ovario era duplo existindo além do normal um outro menor separado do primeiro pela area da espermatheca; mediam respectivamente $0,80 \times$ 0,35 e $0,32 \times 0,18 \mathrm{~mm}$. Anomalia do testiculo semelhante a esta já foi referida por Clemente Pereira (1929), a proposito de uma especie do genero Opisthogonimus.

Desta especie não existe uma bôa descripção na litteratura, visto ser a de Looss baseada em tres especies diversas pelo menos. Existem porém bons desenhos devidos a Looss e a Fuhrmann. Relativamente a evolução supponho existir confusão entre as diversas especies sendo util, agora que é moda o estudo dos cyclos evolutivos, que se faça uma revisão do cyclo das diversas especies européas. No estudo da evolução dos trematodeos 
deve-se abandonar a technica geralmente seguida de procurar as Cercarias ao acaso e com estas se tentar obler o adulto. Para que os cyclos sejam verificados sem riscos de confusão se deverá partir dos adultos e infestar molluscos reconhecidamente não parasitados. Este proceder além de menos empirico é realmente mais facil, dado o numero limitado de especies de molluscos relativamente ao numero de especies de trematodeos e de hospedeiros definitivos.

Trabalhamos no seguinte material, além dos exemplares do Museu Zoologico de Hamburgo, que foram restituidos aquelle modelar Instituto:

No. 6.397-6.404 provenientes do Turquetão Russo colleccionado por Strom, de Rana esculenta ridibunda Pall.

No. 6.405 proveniente Saratow-Wolga, colleccionado por Strom, de Rana escul. ridibunda Pall. No. 6.406-6.409 proveniente de Hamburgo e por nós colleccionado, em Rana esculenta L. No. 6.501 proveniente de Hamburgo e por nós colleccionado, em Rana esculenta L.

Pneumonoeces similis (Looss, 1899).

(Est. LXVI, figs. 5-8).

Distomum variegatum auct. p. p.

Dis'omum variegatum Looss, 1894, p. p. pag. 71 (pl. II, fig. 43 ?).

Haematoleuchus similis Looss, 1899, p. 601.

Distoma similis Looss, 1899 , p. 602 , nec Sonsino, 1896.

Haemaloleuchus similis Stiles, 1902, p. 178.

Haematoleuchus similis Stiles \& Hassall, 1902, p. 20.

Haematoleuchus similigenus Stiles \& Hassall, 1902, p. 20.

Pneumonoeces similis Klein, 1905, p. 61.
Pneumonoeces similis Luehe, 1909, p. 104 (não figura 82).

Pneumonoeces similis Wundsch, 1911, p. 40.

Pneumonoeces similigenus Cort, 1915, p. 203.

Pneumonoeces similis Cort, 1915, p. 203.

Preumonoeces similigenus Nicoll. 1926 , p. 15.

Corpo alongado sub-cylindrico. Cuticula guarnecida de pequenos espinhos. Acetabulo menor que a ventosa oral, préequatorial. Ventosa oral sub-lerminal. Pharynge logo em seguida á ventosa; esophago curto e largo; cecos estendendo-se até perto da extremidade posterior do corpo. Póro genital na area do pharynge, sub-mediano. Bolsa do cirro muito long: e delgada, attinge a zona acetabular; contem cirro, prostata e longa vesicula seminal sinuosa. Testiculos redondos com zonas geralmente afastadas e campos coincidindo parcialmenie, post-equaloriass. Ovario redondo com zona e campo abrangendo a zona e campo acelabulares. Espermatheca post-ovariana, maior que o ovario. Utero constitudo por um ramo descendente com ovos de casca ainda de côr clara; este ramo fórma varias alças entre os testiculos e que ultrapassam a area cecal e dirige-se depois para traz, onde fórma de cada lado do corpo uma grande alça longitudinal extra-cecal, com um comprimento approximadamente igual a quarta parte do comprimento total do corpo e attingindo apenas a parte media da zona do testiculo posterior. O ramo ascendente do utero fórma alças que attingem e ultrapassam as areas cecaes antes de attingir a zona acetabular, depois da zona acetabular fica inteiramente intra-cecal. Ovos na ultima porção do utero de côr castanho escura, quasi negra, operculados. Vitellinos constituidos por volumosos folliculos situados na parte 
mediana do corpo; acima do acetabulo os folliculos vitellinicos invadem a area intra cecal, abaixo da zona cetabular se mantem na area extra-cecal; anteriormen- te ficam muito abaixo da bifurcação intestinal e posteriormente terminam na zona do testiculo anterior. As dimensões desta especie são as seguintes:

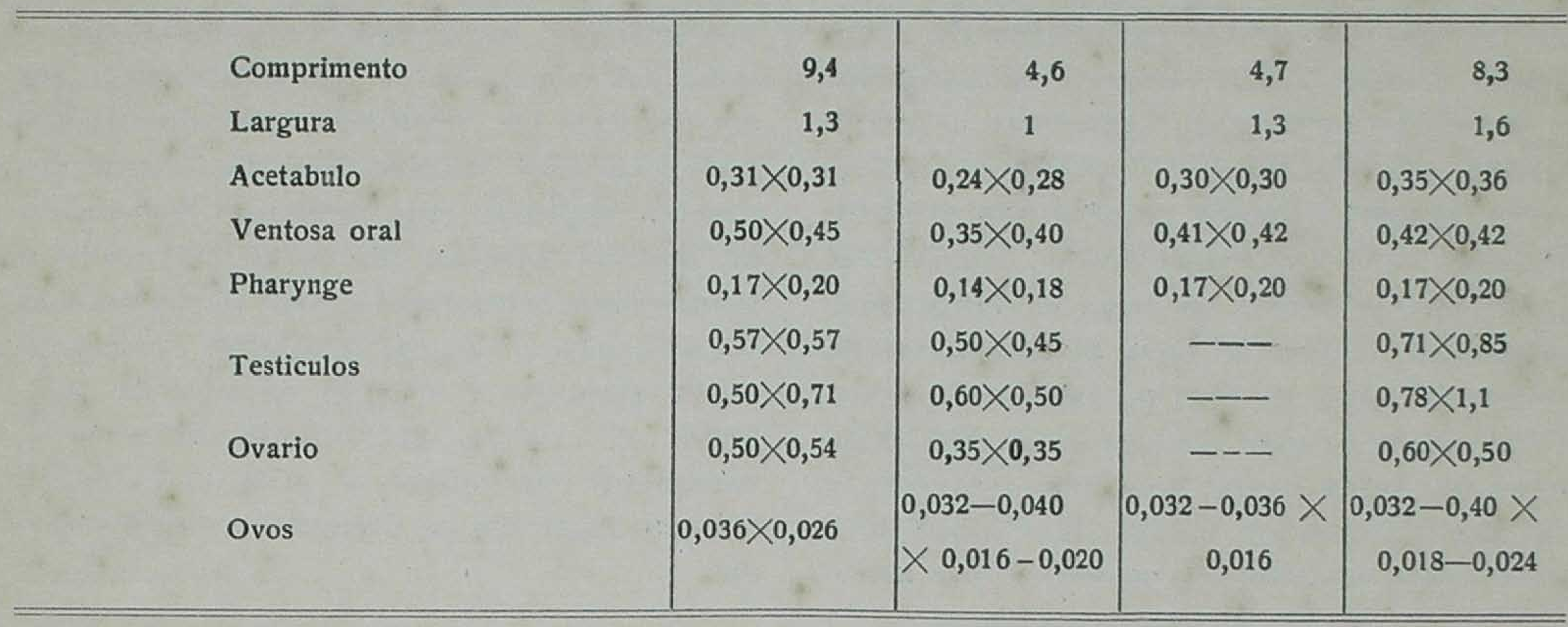

A espermatheca póde ser do tamanho ou maior que o ovario.

Habitat: pulmão de Rana temporaria L., $R$. esculenta L.

Destribuição geographica: Europa.

Esta especie se distingue facilmente de todas as outras especies pela disposição dos vitellinos.

Looss em 1899 diz que a sua figura 43 (1894) corresponde a esta especie, o que nos parece muito duvidoso pela disposição das alças longitudinaes do utero e ainda pela posição dos testiculos. Temos a impressão que a figura de Looss corresponde ao asper no qual não tenha sido representado o vitellino posterior. Esta mesma figura foi reproduzida por Luehe como sendo do $P$. variegatus.

Desta especie trabalhamos no seguinte material, além dos exemplares do Museu Zoologico de Hamburgo:

6.410-6,412. Rana esculenta ridibunda Pall. Saratow-Wolga, col. por M. Strom.

6.412-6417. Rana esculenta L. Hamburgo, col. por Travassos.

6.502. Rana esculenta L. Hamburgo, col. por Travassos.
Pneumonoeces asper (Looss, 1899).

(Est. LXVI, figs. 9-11; est. LXVII, fig. 12).

Distomum variegatum auct. p. p.

Disfomum variegatum Looss, 1894, p. 71 , etc., p. p. (fig. 43, pl. II ?).

Haematoleuchus asper Looss, 1899, p. 601.

Haematoleuchus asper Luehe, 1902, p. 238.

Haematoleuchus asper Stafford, 1902, p. 896.

Pneumonoeces asper Klein, 1905, p. 64.

Pneumonoeces asper Luehe, 1909, p. 104.

Pneumonoeces asper Wundsch, 1911, p. 8, 40.

Haematoleuchus asper Cort, 1915, p. 203.

Pneumonoeces asper Nicoll, 1926, p. 15.

Corpo achatado, de contorno ellipsoide, tendo o polo anterior mais attenuado. Cuticula revestida de pequenos espinhos curtos que lhes empresta aspecto aspero. Acetabulo forte, pré-equatorial e pouco 
menor que a ventosa oral. Ventosa ooral sub-terminal, seguida logo do pharynge que é forte. Esophago curto. Cecos longos e sinuosos, terminando perto da extremidade posterior do corpo. Póro genital na zona do pharynge. Bolsa do cirro longa e com muitas sinuosidades, attinge a zona do acetabulo. Testiculos arredondados, situados na parte média do corpo, com campos e zonas coincidindo parcialmente. Ovario pré-acetabular, lateral, em geral parcialmente na zona e campo acetabulares. Espermatheca geralmente mais volumosa que o ovario, post-acetabular. Utero, com ramo descendente contendo ovos de casca castanho claro, intra-cecal, na parte posterior fórma duas alças longitudinaes lateraes, extra-cecaes que altin- gem, e ás vezes ultrapassam, a zona do testiculo posterior. O ramo ascendente do utero contém ovos de casca escura e quasi negra, dirige-se para dianie na area intra-cecal formando alças ma's ou menos grandes. Acima do acetabulo torna-se mais estreito e fórma um grupo de alças enoveladas. Os vitellinos são constituidos por folliculos volumosos, não formando rosetas typicas; um anterior, localizado na metade anterior do corpo de modo a formar um semicirculo cuja parte central é intra-cecal e as extremidades cecaes; a outra glandula é situada na metade posterior do corpo, geralmente intra-cecal, raramente invadindo a area extra-cecal. São as seguintes as dimensões desta especie:

\begin{tabular}{l|l|l|l}
\hline \hline Comprimento & 5,2 & 5,1 & 4,0 \\
Largura & 1,4 & 1,6 & 1,3 \\
Acetabulo & $0,34 \times 0,32$ & $0,32 \times 0,32$ & $0,24 \times 0,32$ \\
Ventosa oral & $0,38 \times 0,38$ & $0,34 \times 0,44$ & $0,28 \times 0,31$ \\
Pharynge & $0,14 \times 0,17$ & $0,14 \times 0,17$ & $0,12 \times 0,12$ \\
Testiculos & $0,85 \times 0,64$ & $0,85 \times 0,68$ & $0260 \times 0,57$ \\
Ovario & - & $1,1 \times 0,61$ & - \\
Ovos & $0,57 \times 0,42$ & $0,42 \times 0,31$ & $0,44 \times 0,48$ \\
& $0,056 \times 0,028-0,032$ & $0,056-0,064 \times$ & $0,054-0,056 \times$ \\
\hline \hline
\end{tabular}

Habitat: pulmão de Rana esculenta L., R. esculenta ridibunda Pall.

Distribuição geographica: Europa.

Esta especie, creada por Looss como especie dubia, foi bem estudada por Wundsch que fez um detalhado estudo comparativo com o similis.

Desta especie trabalhamos no seguinte material, além de alguns exemplares do Museu Zoologico de Hamburgo:

6.418-6.428. Rana esculentaa L. proveniente de Hamburgo colleccionados por Travassos.

6.427. Rana esculenta ridibunda Pall.
S a r a to $\mathrm{w}-\mathrm{W}$ o lg a col. por Strom.

6.502-6.505. Rana esculenta, Hamburgo, colleccionado por Travassos.

Pneumonoeces longiplexus (Stafford, 1902).

(Est. LXVII, figs. 13-14).

Haematoleuchus longiplexus Stafford, 1902, p. 901, pl. 33, fig. 1.

Pneumonoeces longiplexus Stafford, 1905, p. 687.

Preumonoeces longiplexus $\mathrm{Klein}$, 1905 , p. 64. 
Pneumonoeces longiplexus Seely, 1906, p. 249.

Pneumonoeces longiplexus C ort, 1915 , p. 212, fig. 1E, 4A, 10, 12, 14.

Pneumobites longiplexus Ward, 1917, p. 5.
Pneumobiles longiplexus Ward, 1918, p. 403, fig. 700 .

Pneumonoeces longiplexus I $\mathrm{r} \mathrm{w}$ in, 1929, p. 74.

Desta especie, estudada por Stafford e Cori, resumiremos no quadro abaixo as principaes caracteristicas.

\begin{tabular}{|c|c|c|c|}
\hline * & STAFFORD & & CORT \\
\hline Comprimento & 7 a $8 \mathrm{~mm}$. podendo attingir até $15 \mathrm{~mm}$. & & $2,6 \mathrm{~mm}$ \\
\hline Largura & 2 podendo attingir $3 \mathrm{~mm}$. & & $2 \mathrm{~mm}$. \\
\hline Cuticula & Sem espinhos. & & Com espinhos. \\
\hline Acetabulo & 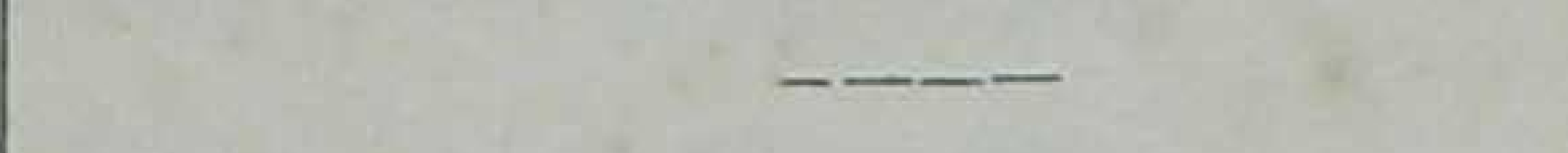 & & $0,17 \mathrm{~mm}$. \\
\hline $\begin{array}{l}\text { Relação entre o } \\
\text { acetabulo e a } \\
\text { ventosa oral }\end{array}$ & - & & $2: 1$ a $5: 3$ \\
\hline Ventosa oral & $0,46-0,7 \mathrm{~mm}$ & & 2 vezes maior que o acetabulo; $0,36 \times 7,46 \mathrm{~mm}$. \\
\hline Pharynge & --- & & $0,22 \times 0,18 \mathrm{~mm}$ \\
\hline Testiculos & Alongados e symetricos. & 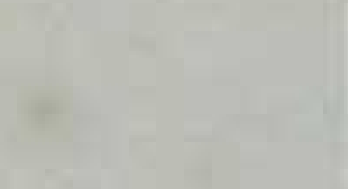 & Alongados e irregulares. \\
\hline Ovario & Ao nivel do acetabulo. & & Lobado, oblongo. \\
\hline Utero & $\begin{array}{l}\text { Com alças longitudinaes attingindo quasi } \\
\text { tosa oral. }\end{array}$ & a ven- & Com alças longitudinaes attingindo o pharynge. \\
\hline Vitellinos & Em toda a extensão do corpo, em roseta. & & $\begin{array}{l}\text { Com } 16 \text { a } 26 \text { folliculos em cada roseta. Em to- } \\
\text { da a extensão do corpo desde logo } \\
\text { abaixo do pharynge até perto da extre- } \\
\text { midade posterior. }\end{array}$ \\
\hline Ovos & Com $0,022 \times 0,017$. & & 0,022 a $0,027 \times 0,014$ a 0,017 . \\
\hline
\end{tabular}
Norte.

Destribuição geographica: America do

Comparando as descripç̃es e figuras destes auctores notamos como unica differença apreciavel a presença de espinhos notada por Cort e que não foram vistos por Stafford.

Como já fizemos notar, não nos parece razoavel a separação desta especie em genero a parte.

Pneumonoeces breviplexus (Stafford, 1902).

(Est. LXVII, figs. 15-16).

Haematoleuchus brcviplexus Stafford,
1902, p. 904, pl. 33, fig. 2 .

Pneumonoeces breviplexus Stafford, 1905 , p. 687.

Pneumonoeces breviplexus Klein, 1905, p. 61.

Pneumonoeces breviplexus Cort, 1915 , p. 216, fig. $1 \mathrm{~F}, 9$.

Pneumonoeces breviplexus W a rd, 1918, p. 403.

Pneumonoeces breviplexus I rwin, 1929 , p. 75.

Esta especie foi estudada por Stafford e Cort de cujas descripções fazemos o resumo abaixo. 


\begin{tabular}{|c|c|c|}
\hline & STAFFORD & CORT \\
\hline Comprimento & 12 a $18 \mathrm{~mm}$. & $5, \mathrm{~S}$ a $9,4 \mathrm{~mm}$. \\
\hline Largura & 2 a $2,5 \mathrm{~mm}$. & 1,3 a 2,7 . \\
\hline Cuticula & Com espinhos dirigidos para traz. & Lisa \\
\hline Acetabulo & $0,12 \times 0,16 \mathrm{~mm}$ & Com metade da ventosa oral. \\
\hline Ventosa oral & Com o dobro do acetabulo. & $0,28 \mathrm{~mm}$. \\
\hline Pharynge & $\therefore$ & $0,14 \mathrm{~mm}$. \\
\hline Testiculos & Obliquos, lobados e alongados. & Obliquos, lobados e alongados. \\
\hline Ovario & Lobado; post-acetabular. & Profundamente lobado. \\
\hline Utero & $\begin{array}{l}\text { Alças longitudinaes não ultrapassando a zona } \\
\text { do testiculo posterior. }\end{array}$ & $\begin{array}{l}\text { Alças logitudinaes attingindo a zona do testiculo } \\
\text { posterior. }\end{array}$ \\
\hline Vitellinos & Em rosetas de 8 a 15 elementos. & Em rosetas de 12 a 20 elementos. \\
\hline Ovos & $0,022 \times 0,17 \mathrm{~mm}$ & 0,020 a $0,026 \times 0,013$ a $0,016 \mathrm{~mm}$. \\
\hline & Rana catesbiana, $R$. virescens & Rana catesbiana, $R$. clamitans, Bufo americanus \\
\hline
\end{tabular}

Destribuição geographica: America do Norte.

Entre as descripções de Stafford e Cort ha a notavel differença da ausencia de espinhos cuticulares provavelmente devidas a um equivoco de Stafford. O ovario tambem apresenta differenças na lobulação, assim como as alças longitudinaes do ulero que vão muito mais adiante nos exemplares de Cort. Não obstante essas differenças nos parece fóra de duvidas a identidade das especies.

Pneumonoeces varioplexus (Stafford, 1902).

(Est. LXVII, figs. 17-20).

Haematoleuchus varioplexus Stafford, 1902, p. 907, fig. 3.

Haematoleuchus similiplexus Stafford, 1902, p. 907, fig. 4.

preumonoeces varioplexus Stafford, 1905 , p. 687.

Pneumonoeces similiplexus Stafford, 1905 , p. 687.

Preumonoeces varioplexus Klein, 1905, p. 61.

Pneumonoeces similiplexus Klein, 1905 , p. 64.
Pneumonoeces similiplexus Se ely, 1906, p. 252.

Pneumonoeces varioplexus $\mathrm{Cort}$, 1915, p. 204.

Pneumonoeces similiplexus $\mathrm{C}$ or $\mathrm{t}$, 1915 , p. 219 , fig. $1 \mathrm{~A}, 3,4 \mathrm{~B}$.

Esta especie foi descripta por Stafford como sendo duas, julgando Cort dever considerar como uma. Acceitamos o criterio deste ultimo. Resumimos aqui os caracteres mencionados por Stafford para os seus $P$. similiplexus e varioplexus e os de Cort.

Consideramos como tendo prioridade a denominacãa de varioplexus, por ter sido debaixo deste nome que primeiro foi descripta no trabalho de Stafford. Reproduzimos tambem as duas figuras de Stafford e a de Cort.

Distribuição geographica: Ameriea do Norte.

A não ser as dimensões das alças uterinas longitudinaes, que na descripção de Cort attingem a zona do lesticulo anterior, tudo o mais concorda de modo evidente. 


\begin{tabular}{|c|c|c|}
\hline & STAFFORD & CORT \\
\hline Comprimento & 3 a $8 \mathrm{~mm}$. e $9 \mathrm{~mm}$ & $5,8 \mathrm{~mm}$ \\
\hline Largura & $2 \mathrm{~mm}$ & $1,9 \mathrm{~mm}$ \\
\hline Cuticula & Com espinhos. & $\begin{array}{l}\text { Com espinhos na porção anterior, adiante dos } \\
\text { testiculos. }\end{array}$ \\
\hline Acetabulo & Grande, pre-equatorial, com 0,38 e $0,41 \mathrm{~mm}$. & Na relação de $3: 4$ para a ventusa anterfor. \\
\hline Ventosa oral & $0,44 \times 0,41 \mathrm{~mm}$. e $0,57 \times 0,51 \mathrm{~mm}$ & Na relação de $4: 3$ para o acetabulo. \\
\hline Pharynge & -7 & $\mathrm{Na}$ relação de $1: 2$ com a ventosa oral. \\
\hline Testiculos & Pequenos, redondos, em geral regulando o ovario & Sem lobos, redondos ou ovaes. \\
\hline Ovario & Redondo, abaixo do acetabulo. & Sem lobos, redondo ou oval. \\
\hline Utero & $\begin{array}{l}\text { Com alças longitudinaes attingindo a zona do } \\
\text { testiculo posterior. }\end{array}$ & $\begin{array}{l}\text { Com alças longitudinaes attingindo a zona do } \\
\text { testiculo anterior. }\end{array}$ \\
\hline Vitellinos & Com rosetas. & $\begin{array}{l}\text { Com rosetas de } 6 \text { a } 13 \text { foliculos e dispostas des- } \\
\text { de logo abaixo do esophago até a ex- } \\
\text { tremidade posterior. }\end{array}$ \\
\hline Ovos & Com $0,039 \times 0,019$ & 0,034 a $0,040 \times 0,017$ a $0,021 \mathrm{~mm}$. \\
\hline Habitat & Rana virescens, Bufo lentigiosus. & Rana pipiens, Bufo americanus. \\
\hline
\end{tabular}

Pneumonoeces schulzei Wundsch, 1911.

(Est. LXVIII, fig. 21).

\section{Pneumonoeces schulzei Wundsch, 1911, p. $38,41$.}

Desia especie não existem descripção detalhada nem figuras. Limitou-se Wundsch apenas a salientar as differenças entre ella e as outras especies. No material do Museu Zoologico de Hamburgo encontramos um exemplar de Pneumonoeces que julgamos corresponder a especie de Wundsch. Infelizmente este exemplar não estava bem conservado, além de estar muito retrahido. Deste exemplar damos a descripãção que segue e um desenho.

Corpo ellipsoide, tendo o polo anterior mais attenuado; mede $4,6 \mathrm{~mm}$. de comprimento por $1,7 \mathrm{~mm}$. de largura maxima. Cuticula revestida de espinhos pequenos. Acetabulo forte, tão grande como a ventosa oral, mede $0,35 \mathrm{~mm}$. de diametro. Ventosa oral sub-terminal com cerca de $0,25 \mathrm{~mm}$. de diametro longitudinal por $0,41 \mathrm{~mm}$. de diametro transversal.
Pharynge logo em seguida á ventosa oral, mede 0,14 de diametro longitudinal por $0,18 \mathrm{~mm}$. de diametro transversal. Cecos longos e amplos, vão até perto da extremidade posterior do corpo. Póro genital ao nivel, do pharynge. Bolsa do cirro longa e sinuosa, attinge a zona acetabular. Testiculos ellipsoides, alongados no sentido do eixo longitudinal do corpo, com zonas coincidindo quasi inleiramente e campos apenas em contacto. medem respectivamente $1,21 \times 0,85$ e $1,21 \times 0,78 \mathrm{~mm}$. Ovario pré-equatorial. lateral, com a area coincidindo parcialmente com a area acetabular, mede cerca de 0,62 por $0,35 \mathrm{~mm}$. de diametro. Espermatheca na zona do ovario. Utero com ramo descendente formando alças na area intra-cecal e na parte posterior formando alças longitudinaes extra-cecaes que attingem e ultrapassam as zonas testiculares. Estas alças são irregulares e formam muitas sinosidades. Porção as cendente do utero na area intra cecal; na zona acetabular e acima a utero fórma alças apertadas e enoveladas que algu- 
mas vezes ultrapassam a area cecal. Ovos de casca escura quasi negra, como nas outras especies, medem cerca de 0,032 a 0,035 por 0,016 a $0,018 \mathrm{~mm}$.

Vitellinos constituidos por acnes formando rosetas; um anterior e oulro posterior formando semicirculos de concavidades voltadas para o meio do corpo; a parte central dos semicirculos dos vitellinos é situada na area intra-cecal e os extremos ficam na area cecal e extracecal.

\section{Habitat: pulmão de Rana sp.}

Disiribuição geographica: Europa.

E' a seguinte a diagnose de Wundsch :

P. schulzei-Pelle com espinhos. Corpo regularmente oblongo, com 15 a 18 mm. de comprimento. Ramos da vesicula excretora ultrapassando claramente o acetabulo. Ventosas relativamente grandes. Ovario redondo. Testiculos ovaes e com margens regulares. Vitellinos attingindo a parte posterior do corpo e com grupos de folliculos formando rosetas typicas. Ovos com 0,025 a 0,027 por $0,019 \mathrm{~mm}$. Esta diagnose se afasta da nossa descripção pelo tamanho do parasito, mas o exemplar que examinamos estava muito contrahido, aliás os trematodeos são suscepliveis de grandes variações no seu tamanho.

Esta especie se approxima bastante, pela anatomia geral, do $P$. asper, deste porém se distingue facilmente pelas dimensões dos ovos e pela disposição dos folliculos vitellinicos em rosetas typicas. Pelas dimensões dos ovos se approxima do $P$. similis do qual se distingue logo pelos vitellinos e pelas dimensões relativas das ventosas.

Pneumonoeces campyristis Klein, 1905.

(Est. LXVIII, fig. 22).

Pneumonoeces campyristis Klein, 1905 , p. 60 , pl. 5 , figs. $1-2$.

Pneumonoeces campyristis Johnston, 1912 , p. 325.
Pneumonoeces campyristis Cort, 1915, p. 205.

Resumimos aqui a descripção de Klein para esta especie.

Comprimento $6 \mathrm{~mm}$; largura 1,4 mm.; cuticula sem espinhos; acetabulo com $0,15 \mathrm{~mm}$; ventosa oral com 0,32 mm.; pharynge com $0,18 \mathrm{~mm}$; bolsa do cirro curta, não attingindo o acetabulo, mede $0,84 \mathrm{~mm}$. de comprimento por 0,24 $\mathrm{mm}$. de largura; testiculos alongados, com campos e zonas coincidindo parcialmente; ovario na zona acetabular; utero com alças longitudinaes não ultrapassando as zonas testiculares; ovos com 0,028 a 0,030 por 0,014 a $0,018 \mathrm{~mm}$.; viiellinos, com rosetas atypicas em numero de 6 a 8 e lconstituidas por 4 a 8 folliculos, um na porção anterior e outro na porção posterior do corpo, na area intra e extra-cecal.

Habitat: pulmão de Rana exadactyla Less.

Distribuição geographica: India.

Pneumonoeces australis Johnston, 1912.

(Est. LXVIII, fig. 23).

Pneumonoeces australis Johnston, 1912 , p. 296, 320, fig. 11, 56-62.

Pneumonoeces australis Cort, 1915, p. 205.

Damos o resumo da descripção de Johnston.

Corpo em fórma de lingua; comprimento 3,5 a $4,9 \mathrm{~mm}$.; largura $1,5 \mathrm{~mm}$.; cuticula lisa, sem espinhos; acetabulo com $0,19 \mathrm{~mm}$., na proporção de $1: 2 \mathrm{com}$ i ventosa oral; ventosa oral com $0,39 \mathrm{~mm}$.; pharynge do tamanho do acetabulo; bolsa do cirro muito longa e tubular, attingindo o ovario; testiculos quasi na mesma zona; ovario na zona acetabular, lobado; utero com alças longitudinaes ultrapassando as zonas testiculares; ovoscom 0,020 por $0,014 \mathrm{~mm}$; vitellinos formando um grupo anterior e outro pos- 
terior de typicas rosetas de 12 a 15 folliculos, na area extra-cecal e intra-cecal.

Habitat: pulmão de Hyla aurea e Limnodynasier peroni.

Distribuição geographica: Australia.

Pneumonoeces noivai Travassos \& Artigas, 1927.

(Est. LXVIII, figs. 24-25; est. LXIX, fig. 26 ; est. LXX, figs. $27-28$ ).

Pneumonoeces neivai Travassos \& Artigas, 1927, p. 212, fig. I.

Pneumonoeces planorbinus $\mathrm{Lutz}$, 1928 , p. 108.

Pneumonoeces pseudis Lutz, 1928, p. 133, fig. 3, est. 25 .

Corpo de fórma oblonga, com um comprimento que varia de 3 a $7 \mathrm{~mm}$. por uma largura maxima de 1,3 a 2,5 $\mathrm{mm}$. Cuticula lisa. Acetabulo ausente, não tendo sido observados mesmo em córtes histologicos. Ventosa oral sub-terminal, medindo de 0,32 a $0,55 \mathrm{~mm}$. de diametro. Pharynge em seguida á ventosa oral, espherico e medindo 0,12 a $0,25 \mathrm{~mm}$. de diametro. Esophago muito curto. Cecos amplos, geralmente repletos de sangue (fig. 27) e se estendendo até perto da extremidade posterior do corpo e em grande parte occultos pelas alças uterinas. Póro genital junto á ventosa oral, mediano. Bolsa do cirro muito longa e sinuosa, geralmente muito difficil de observar em preparados totaes; attinge a zona ovariana; contém uma longa vesicula seminal sinuosa (fig. 26). Testiculos equatoriaes ou post-equatoriaes, com zonas coincidindo quasi inteiramente e campos em contacto, são alongados no sentido longitudinal e apresentam fraca lobulação; medem cerca de 0,72 a $1,2 \mathrm{~mm}$. de comprimento por 0,4 a $0,8 \mathrm{~mm}$. de largura maxima. Ovario redondo, mediano, pre-equatorial, mede cerca de 0,30 a $0,55 \mathrm{~mm}$. de diametro. Glandula de Mehlis muito desenvolvida, situada entre o ovario e os testiculos e de contornos pouco nitidos.
Espermatheca muito grande, entre o ovario e os testículos. Utero com um ramo descendente sinuoso e passando entre os testiculos; alcas longitudinaes extra-cecaes muito desenvolvidas, ultrapassando a zona ovariana; ramo ascendente do utero formando numerosas alças sobretudo acima da zona ovariana. Ovos de casca castanha escura, de operculo pouco nitido, medem de 0,053 a $0,061 \mathrm{~mm}$. de comprimento por $0,030 \mathrm{~mm}$. de maior largura. Vitellinos de folliculos volumosos e não formando rosetas typicas; um situado anteriormente na area intra e extra-cecal e outro situado posteriormente na area intra-cecal.

Habitat: pulmão de Leptodactylus ocellatus L. Lutz refere o seu $P$. pseudis, ao que parece, ao Pseudis paradoxa (L.) da Venezuela.

Distribuição geographica: Rio de Janeiro, S. Paulo-Brasil e Venezuella.

O $P$. neivai se localisa no pulmão destruindo largamente o parenchyma e algumas vezes reduzindo-o a ponto de tornal-o uma fina membrana constituida pela pleura visceral (figs. 27 e 28 ). Ficam sempre com a extremidade anterior dirigida para a pleura e com a ventosa oral commumente em contacto com ella. Sua nutrição é em grande parte feita de sangue que póde ser observado enchendo inteiramente os cecos onde os globulos vermelhos são completamente nitidos, sobretudo na porção mais anterior onde são menos dirigidos (fig. 27).

Sobre a evolução deste parasito Lutz diz a proposito de seu $P$. planorbinus a pagina 107: "Puse en claro la biologia de esta especie cuyas partenitas viven en varias especies grandes de Planorbis. Los sporocystos son pequenos e redondeados e las cercarias no tienen muchos distintivos, a no ser un grupo de glandulas cefalicas de cada lado do acetabulo. Estas larvas penetran en Agrionidae». Diz ter obtido fórmas jovens com cercaria de Limnaea que admitte sejam de Haplome 
tra palmipedis Lutz, 1928 (=Glypthelmins) ou de Pneumonoeces. E' interessante que as cercarias attribuidas a esta especie por Lutz apresentem acetabulo, orgão que falla no adulto, aliás se póde conservar rudimentar.

Pneumonoeces parviplexus Irwin, 1929.

$$
\text { (Est. LXIX, fig. 29). }
$$

Pnenmonoeces parviplexus I $\mathrm{r}$ win, 1929, p. 74, fig. 1, pl. 12.

E’ o seguinte o resumo da descripção desta especie:

Comprimento 3,9 a $8,4 \mathrm{~mm}$. Largura 0,85 a $1,6 \mathrm{~mm}$. Cuticula inteiramente revestida de espinhos. Acetabulo na proporção de $1: 4$ relativamente á ventosa oral, na proporção de 4:1 para o acetabulo e 3:1 para o pharynge. Testiculos alongados e situados obliquamente. Ovario lobado, na zona acetabular. Utero com alças longitudinaes ultrapassando a zona do testiculo posterior. Ovos com 0,023 a 0,029 por 0,015 a $\mathbf{0 , 0 1 9} \mathbf{m m}$. Vitellinos formando cerca de 31 rosetas de 20 a 40 folliculos.

Habitat: pulmão de Rana clamitans.

Distribuição geographica: Norte America.

\section{Pneumonoeces fuellebor ni $\mathrm{n}$. sp.}

\section{(Est. LXXI, fig. 30).}

Desta especie apenas obtivemos um exemplar, mas é tão caracteristica que permitte uma bôa definição.

Comprimento $10 \mathrm{~mm}$.; largura 2,3 $\mathrm{mm}$. Cuticula lisa. Corpo claviforme com a maior largura post-equatorial. Acetabulo bem desenvolvido, equatorial, mede $0,31 \mathrm{~mm}$. de diametro longitudinal por $0,37 \mathrm{~mm}$. de diametro transversal. Ventosa oral sub-terminal, grande, mede 0,67 $\mathrm{mm}$. de diametro. Pharynge logo em seguida á ventosa oral, forte, mede 0,30 $\mathrm{mm}$. de diametro. Esophago relativamente curto. Cecos longos e pouco lar- gos, estendem-se até a extremidade posterior do corpo. Póro genital na zona do pharynge; bolsa do cirro muito longa e sinuosa contendo um cirro nitido, prostata e longa vesicula seminal, estende-se até muito perto do acetabulo, isto é, até perto do meio do corpo. Testiculos alongados no sentido longitudinal, relativamente pequenos, post-equatoriaes, com zonas e campos em contacto; medem os testiculos respectivamente 0,57 e $0,71 \mathrm{~mm}$. de comprimento por 0,57 e $0,42 \mathrm{~mm}$. de largura. Ovario alongado longitudinalmente, lateral, parcialmente na zona acetabular, mede 0,81 por $0,50 \mathrm{~mm}$. Espermatheca grande, mediana, parcialmente na zona do ovario, entre este e o testiculo anterior. Utero formando numerosas alças transversaes tanto na metade posterior como na metade anterior do corpo; o ramo descendente e o ascendente são completamente entrelaçados e difficeis de distinguir, as alças longitudinaes são muito reduzidas não attingindo nem a zona do testiculo posterior; o ramo ascendente fórma muitas alças transversaes que ulirapassam a area cecal. $O$ conjuncto do utero lembra, quando observado com pequeno augmento, o typo observado no genero Osíolum, mas não ha formação de grupos de alças transversaes descendentes e ascendentes como naquelle grupo. Vitellinos dispostos em dois grupos, um anterior e outro posterior, existindo porém muitos folliculos em todo o campo lateral mais ou menos occultos pelas alças do utero; anteriormente os vitellinos ficam muito abaixo da bifurcação intestinal intra e extra-cecaes: na parte média do corpo ficam sómente na area extra cecal e finalmente na parte posterior ficam em grande parte na area intra cecal, muito abaixo do testiculo posterior, mas acima da zona da terminação das alças longitudinaes do utero. Os ovos são como nas outras especies de côr escura quasi negra, operculados e medem de 0,032 a $0,039 \mathrm{~mm}$. de comprimento por 0,016 a $0,021 \mathrm{~mm}$. de maior largura. 
Habitat: pulmão de Bufo marinus (L.).

Proveniencia: Estado de S. Paulo.

O unico exemplar desta especie que examinamos foi colleccionado, durante os trabalhos praticos do laboratorio de parasitologia da Faculdade de Medicina de S. Paulo, pelo estudante Nelson Planet.

Sobre as affinidades desta especie já nos referimos no principio do presente trabalho.

Ostiolum Pratt, 1902.

Ostioloum Pratt, 1902, p. 888.

Ostiolum Pratt, 1903, p. 34.

Ostiolum Cort, 1915, p. 228.

Pneumonoeces Ward, 1918, p. 403, p. $p$.

Diagnose: Plagiorchidae: Saphredinae. Trematodeos de tamanho médio, de cuticula lisa ou com espinhos, de acetabulo reduzido; bolsa do cirro muito longa, com cirro, prostata e vesicula seminal; testiculos intracecaes, redondos, com zo. nas e campos em geral coincidindo parcialmente; utero com ramo descendente formando alças transversaes regulares, intra cecaes e nunca formando alçás longitudinaes; ovos de casca escura quasi ne- gra; vitellinos com folliculos de dimensões médias, quasi inteiramente extra-cecaes e na parte média do corpo, formando rosetas.

Habitat: pulmão de anuros.

Especie typo: O. medioplexus (Stafford, 1902).

Ostiolum medioplexus (Stafford, 1902).

(Est. LXXI, figs. 31-33).

Haematoleuchus medioplexus Stafford, 1902, p. 908, pl. 33, fig. 5.

Ostiolum formosum Pratt, 1903, p. $34, \mathrm{pl}$. IV, figs. 6-8.

Pneumonoeces medioplexus Stafford, 1905, p. 687.

Ostiolum formosum Stafford, 1905, p. 687.

Pneumonoeces medioplexus Klein, 1905 , p. 61.

Pneumonoeces medioplexus Seely, 1906, p. 252.

Pneumonoeces medioplexus $\mathrm{Cor}$, 1905, p. 223, fig. 1D, 2, 4C, 11, 13.

Pneumonoeces medioplexus Ir win, 1929, p. 74, 76.

Resumimos em seguida as descripções de Stafford e Cort.

\begin{tabular}{|c|c|c|}
\hline & STAFFORD & CORT \\
\hline Comprimento & 11 a $16 \mathrm{~mm}$. & $7,8 \mathrm{~mm}$. \\
\hline Largura & $1,2 \mathrm{~mm}$. & $1,2 \mathrm{~mm}$. \\
\hline Cuticula & Com espinhos. & Com pequenos espinhos. \\
\hline Acetabulo & $\begin{array}{l}\text { Muito pequeno e difficil de observar, no terço } \\
\text { anterior do corpo, mede } 0,12 \text {. }\end{array}$ & $\begin{array}{l}\text { Na proporção de } 1: 4 \text { com a ventosa oral, mede } \\
0,08 \mathrm{~mm} \text {. }\end{array}$ \\
\hline Ventosa oral & Com $0,31 \mathrm{~mm}$. & Com $0,40 \times 0,34$ a $0,39 \times 0,37 \mathrm{~mm}$ \\
\hline Pharynge & 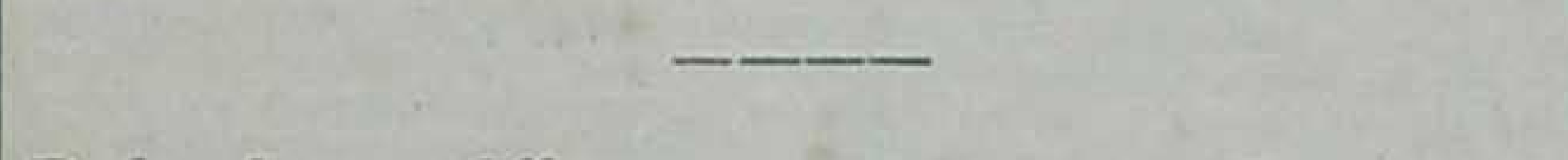 & Com $3 / 4$ da ventosa oral; $0,29 \times 0,26 \mathrm{~mm}$. \\
\hline Testiculos & Redondos e obliquos. & Sem lobos. \\
\hline Ovario & Redondo, post-acetabular. & Sem lobos. \\
\hline Utero & Intra-cecal, sem alças longitudinaes. & Intra-cecal, sem alças longitudinaes. \\
\hline Ovos & Com $0,028 \times 0,018 \mathrm{~mm}$. (Pratt $0,039 \times 0,017)$ & 0,022 a $0,032 \times 0,013$ a $0,018 \mathrm{~mm}$ \\
\hline Vitellinos & Com rosetas de 20 folliculos. & Rosetas de 18 a 23 folliculos grandes. \\
\hline Habitat & Rana virescens, Bufo lentigiosus & Rana pipiens. \\
\hline
\end{tabular}




\section{Norte.}

Desíribuição geographica: America do Ostiolum complexus (Seely, 1906). (Est. LXXI, fig. 34).

Pneumonoeces complexus Seely, 1906, p. 249, figs. 1-2.

Pneumonoeces complexus Cort, 1915, p. 229 , fig. $1 \mathrm{C}$. Seely:

E' este o resumo da descripção de

Corpo allongado com 5 a $8 \mathrm{~mm}$. de comprimenio, por 1,7 a $2 \mathrm{~mm}$. de largura; cuticula sem espinhos; acetabulo com $0,38 \mathrm{~mm}$. de diametro (na proporção cão de $3: 4$ com a ventosa oral); ventosa oral com $0,40 \mathrm{~mm}$; testiculos post-equatoriaes sem lobos; ovario na zona acetabular em parte, alongado; canal de Laurer ausente; utero sem alças longitudinaes, intra cecal; ovos com 0,029 a 0,035 por 0,014 a $0,020 \mathrm{~mm}$; vitellinos desde logo abaixo do pharynge até o fim das zonas testiculares, cada roseta com 6-20 folliculos. piens.

Habitat: pulmão (bocca) de Rana pi-

\section{Explicação das figuras}

\section{ESTAMPA LXVI}

Fig. 1-Eschema de Preumonoeces.

Fig. 2-Eschema de Osliolum.

Fig. 3-Pneumonoeces variegatus-original.

Fig. 4-Pneumonoeces variegatus-ex plar anomalo com duplicação do ovario e dos testiculos.

Fig. 5-Pneumonoeces similis-original: material do Turquestão.

Fig. 6-Pneumonoeces similis-original; material de Hamburgo.

Fig. 7-Pneumonoeces similis-extremidade anterior da fig. 6 .
Distribuição geographica: America do Norte.

Ostiolum coloradensis (Cort, 1917).

(Est. LXXI, figs. 35-36).

Pneumonoeces coloradensis $\mathrm{C}$ ort, 1917 , p. 231, fig. $1 \mathrm{~B}, 4 \mathrm{D}, 5-8$.

Pneumonoeces coloradensis W a $\mathrm{rd}$, 1918, p. 403, fig. 699.

Resumimos a descripção de Cort.

Corpo delgado e longo, com 3,3 a $8,1 \mathrm{~mm}$. de comprimento por 0,5 a 1,5 $\mathrm{mm}$. de largura. Cuticula com espinhos na extremidade anterior. Acetabulo na proporção de 4:5 da ventosa anterior; ventosa oral na proporção de 5:4 do acebulo e de $8: 5$ a $5: 4$ do pharynge; testiculos e ovario redondos, ou ovaes, ou irregulares, sem lobos; utero sem alças longitudinaes extra-cecaes; ovos com 0,032 a 0,039 por 0,018 a $0,021 \mathrm{~mm}$; vitellinos desde o meio da distancia entre o acetabulo e a extremidade anterior do corpo, até perto da extremidade posterior, lateraes, cecaes e extra-cecaes e com 8 a 14 folliculos em cada roseta.

Habitat: pulmão de Rana pipiens.

Distribuição geographica: America do Norte.

Fig. 8-Pneumonoeces similis-original; material de Hamburgo.

Fig. 9-Pneumonoeces asper - original: material do Turquestão.

Fig. 10-Pneumonoeces asper - original; material de Hamburgo.

Fig. 11-Pneumonoeces asper-extremidade anterior da fig. 10 .

\section{ESTAMPA LXVII}

Fig. 12-Pneumonoeces asper - original; material de Hamburgo.

Fig. 13-Pneumonoeces longiplexus - segundo Stafford. 


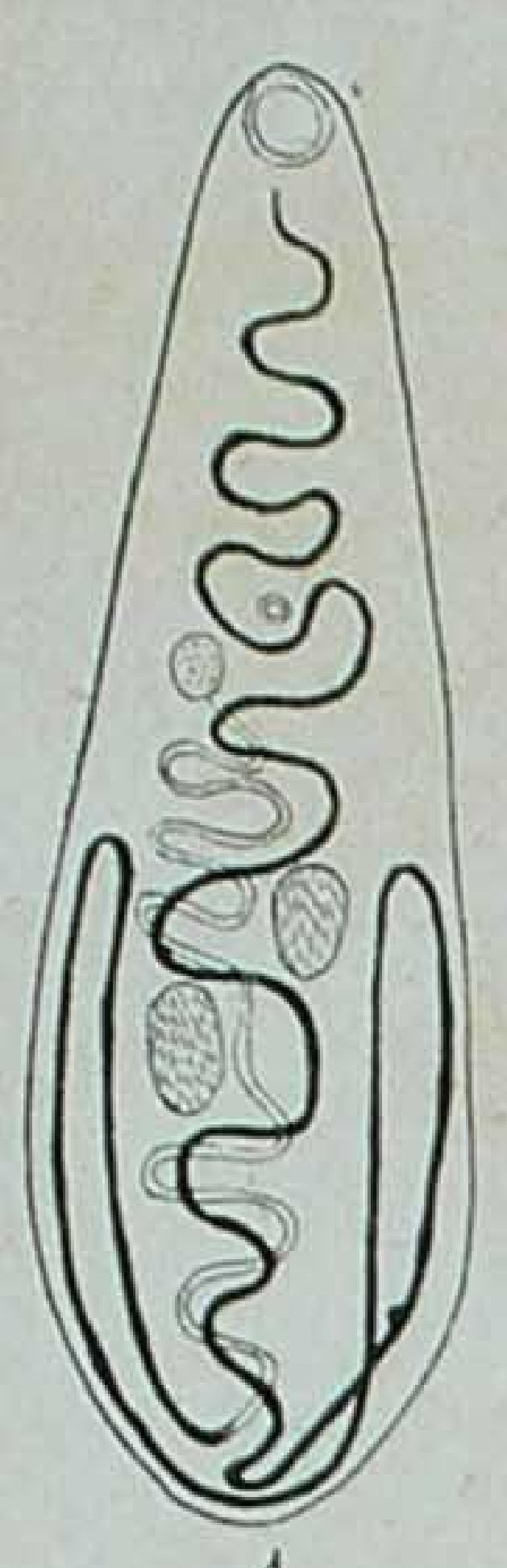

1
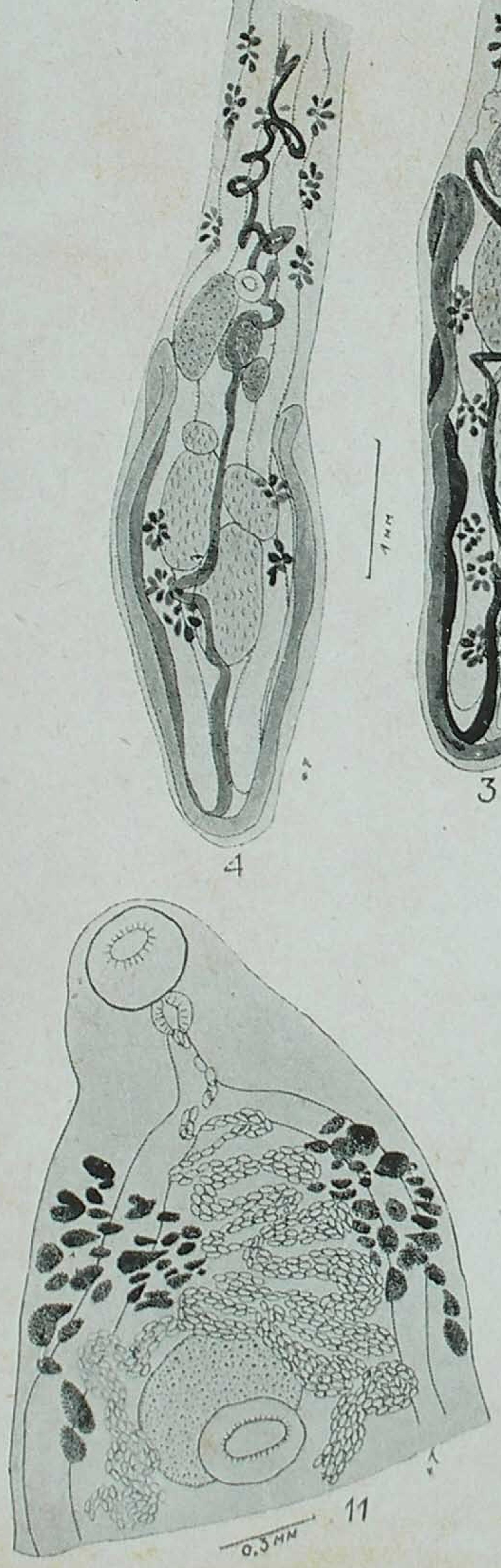

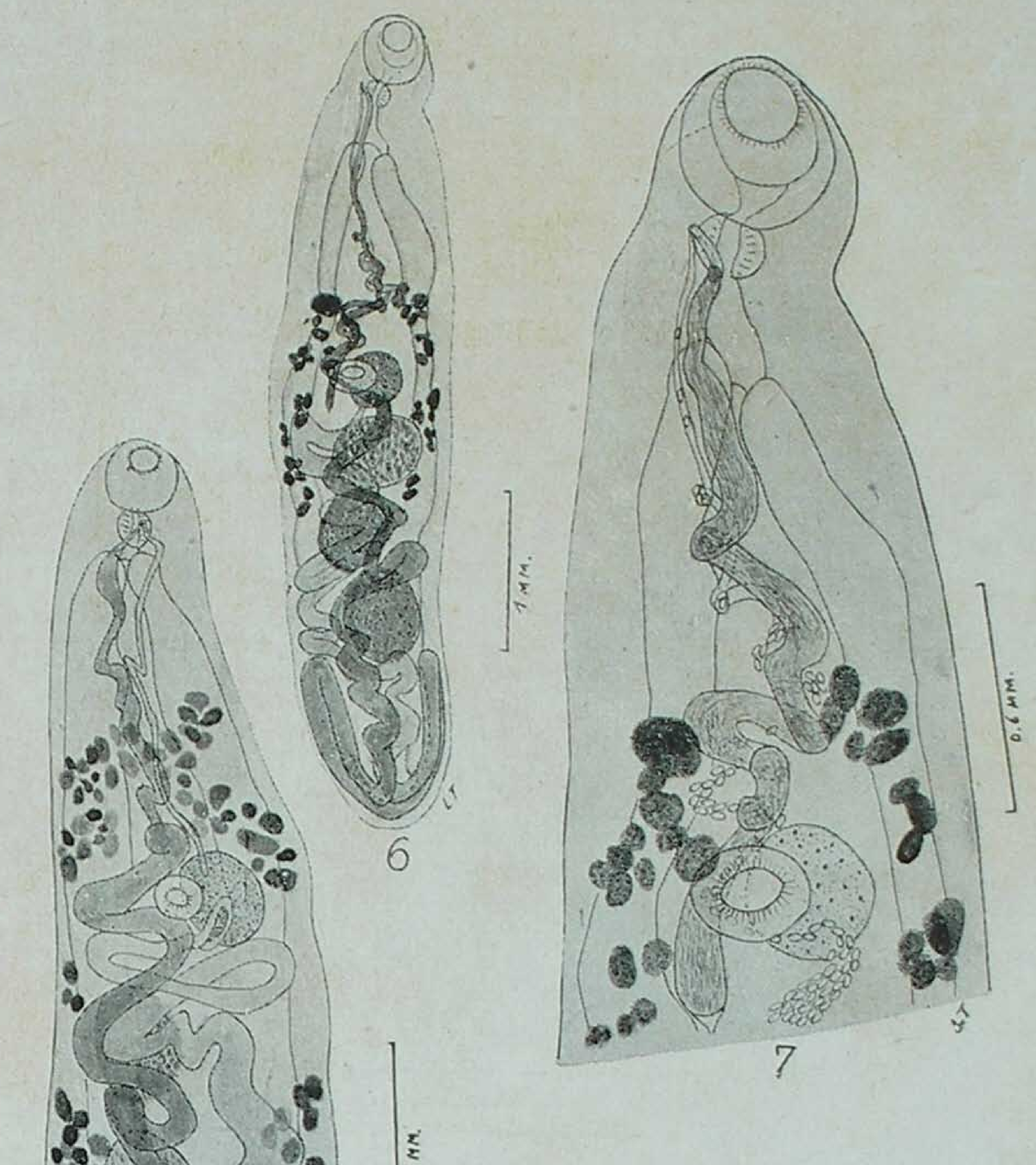

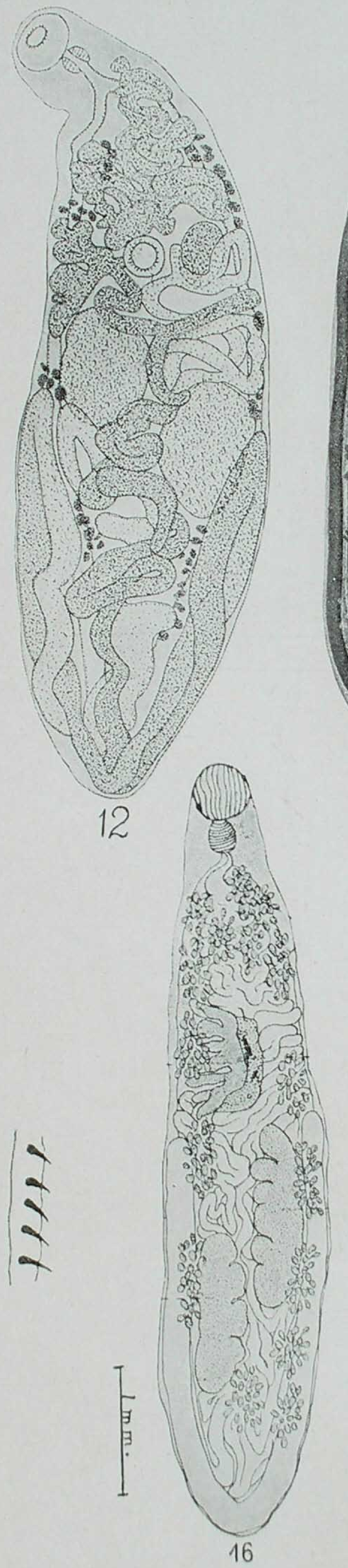
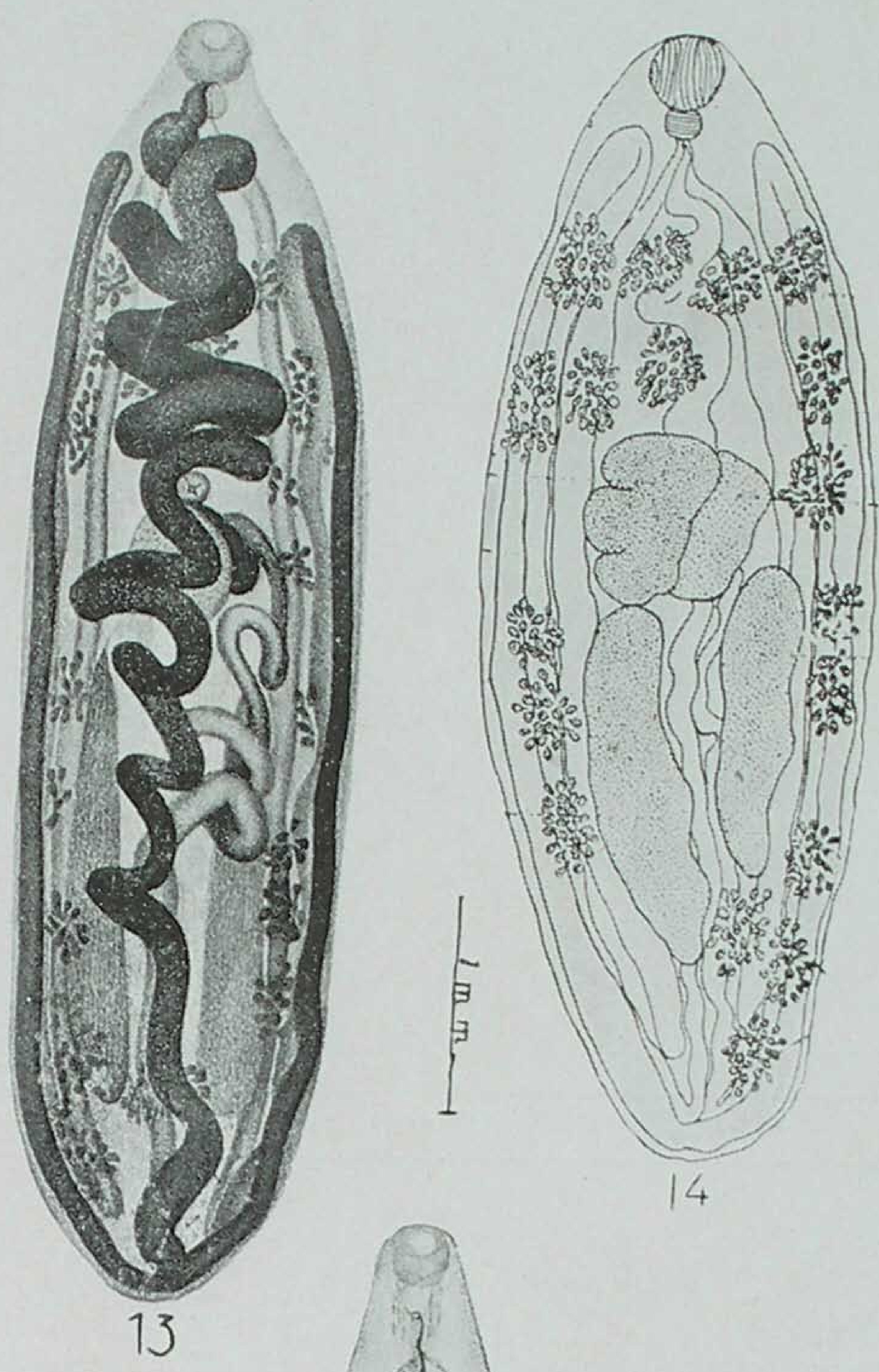

1. है है

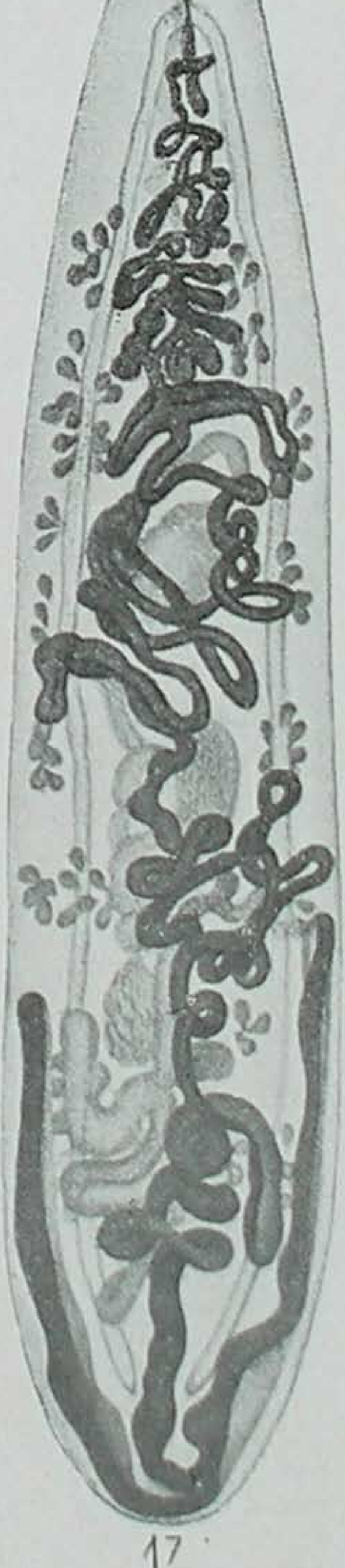

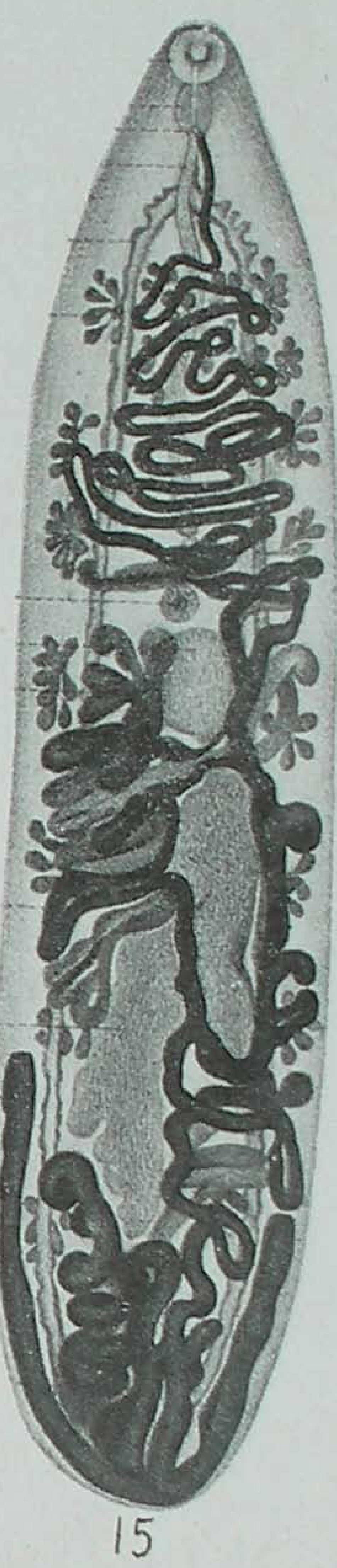

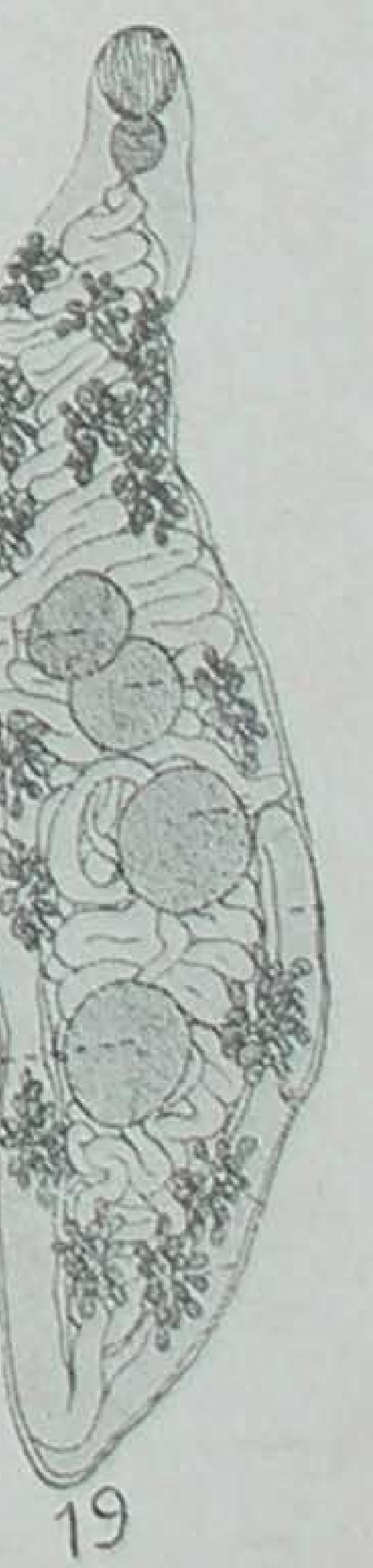



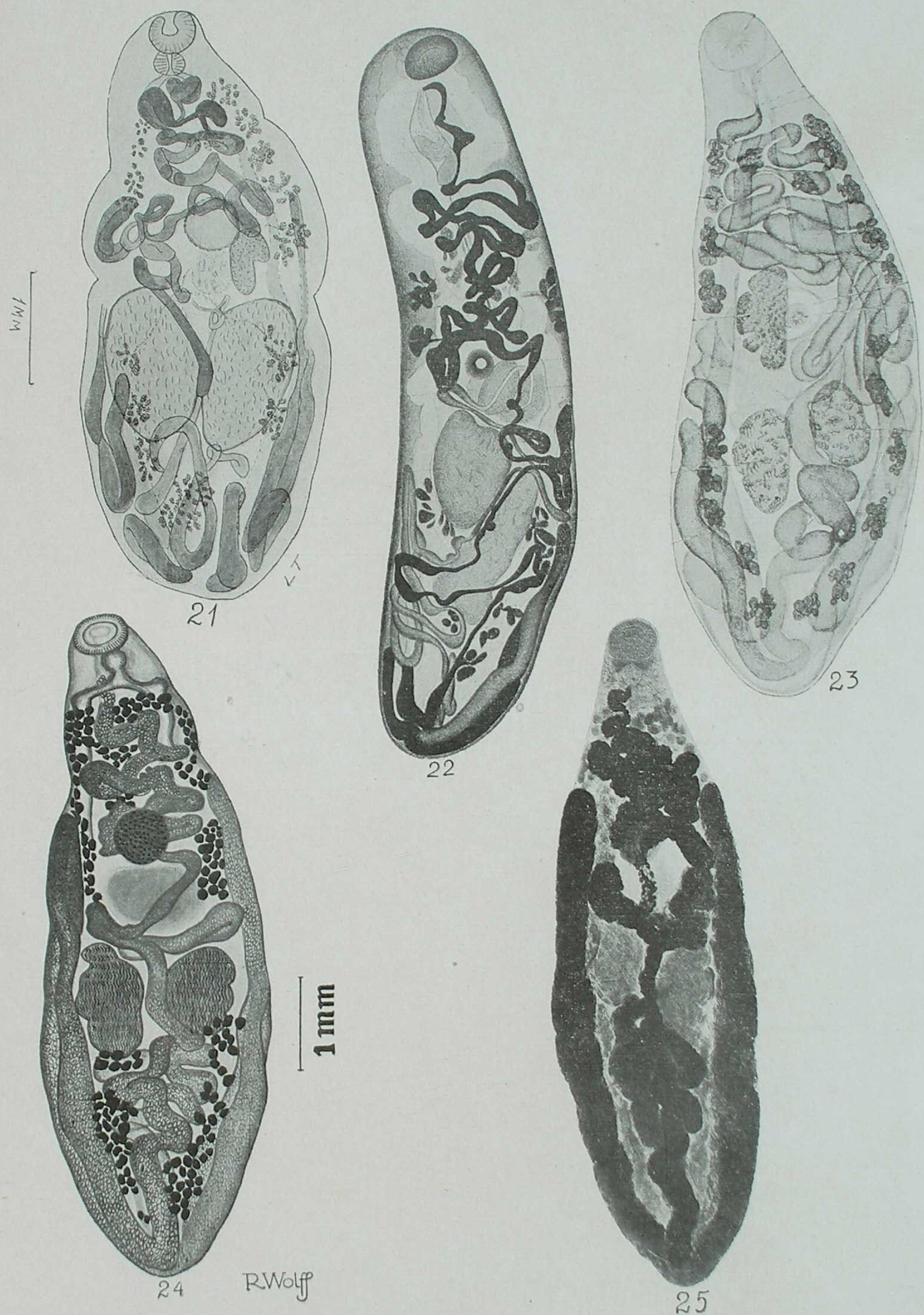

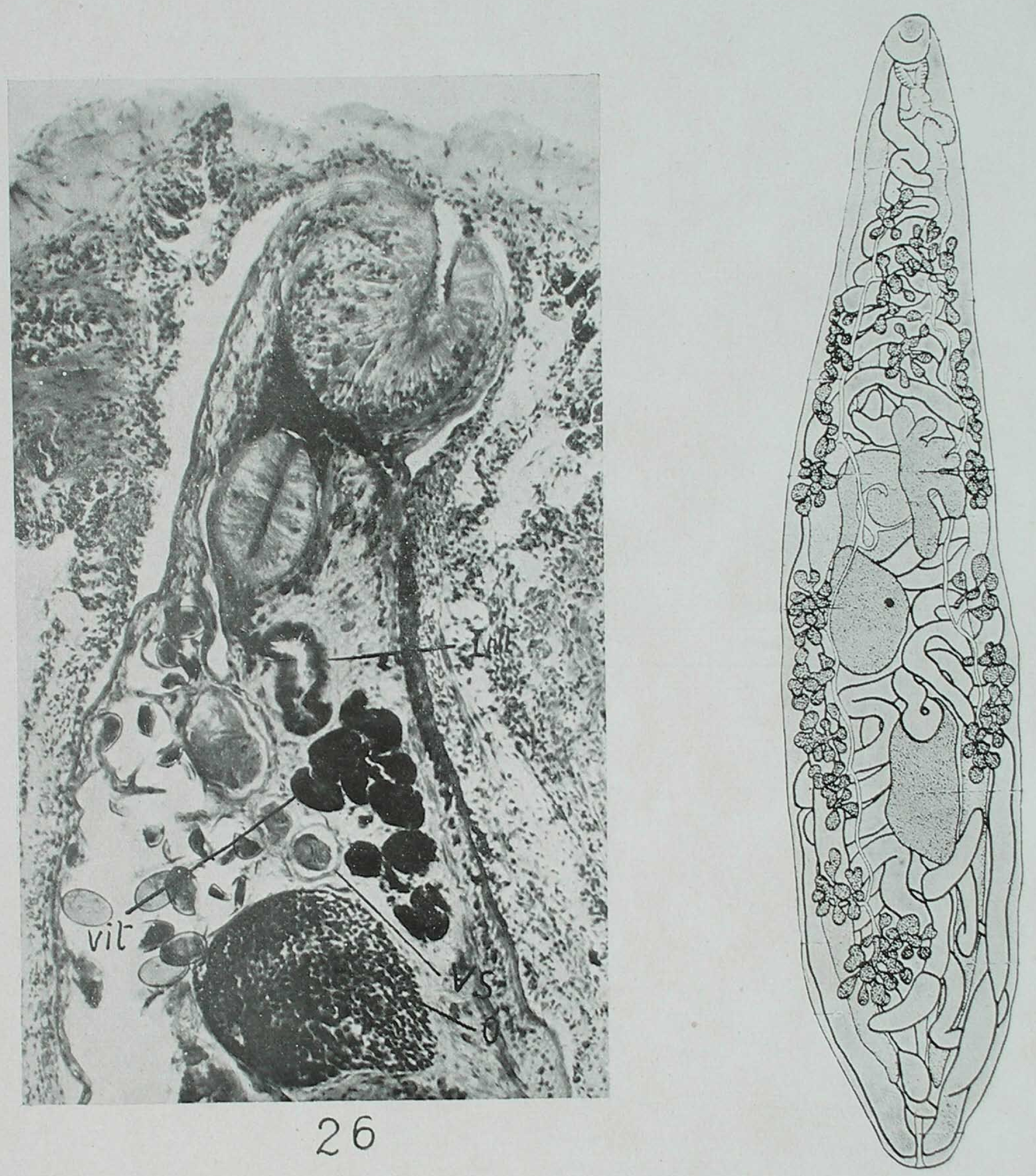

29

L. Travassos e A. R. Darriba: Trematodeos dos generos Pneumonoeces e Ostiolum. 

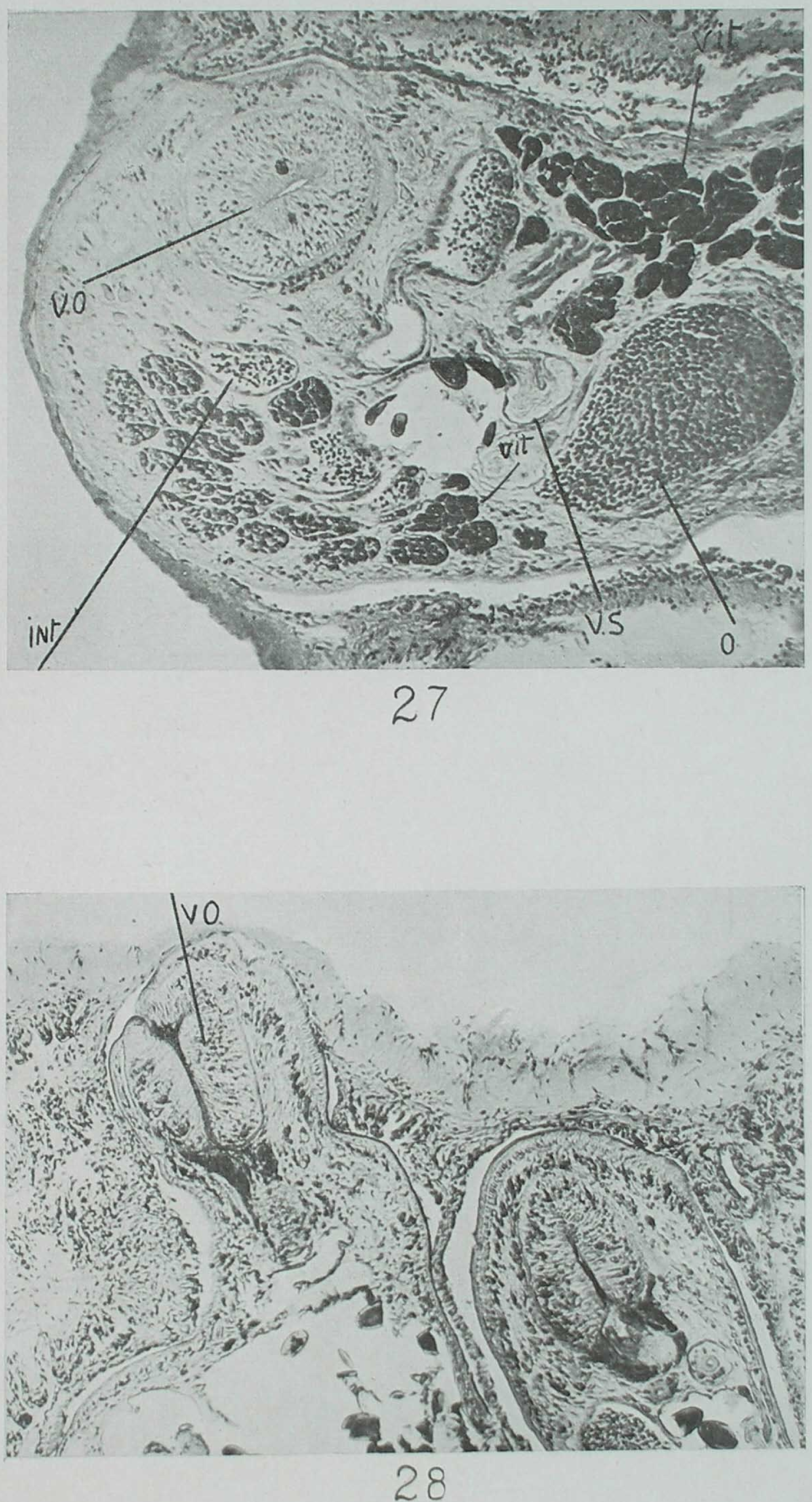

L. Travassos e A. R. Darriba: Trematodeos dos generos Pneumonoeces e Ostiolum. 

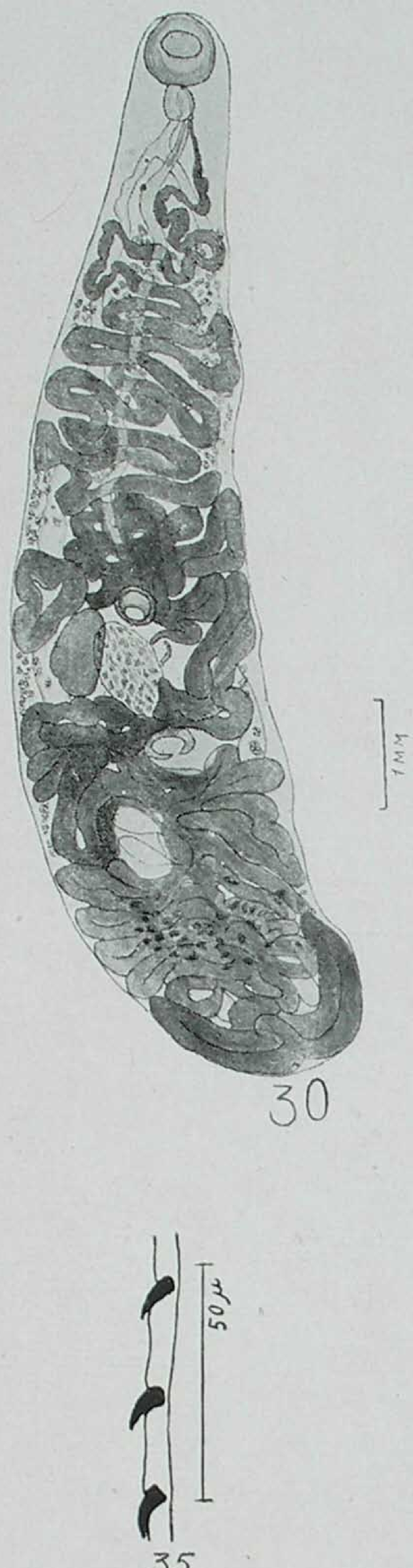

35

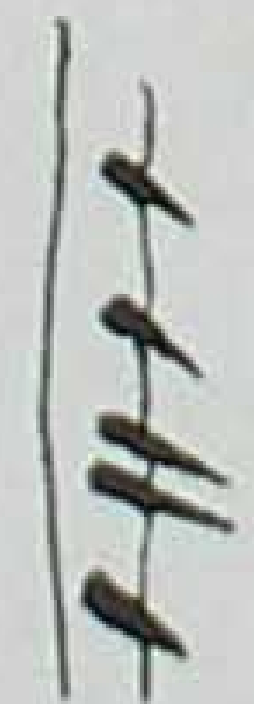

31
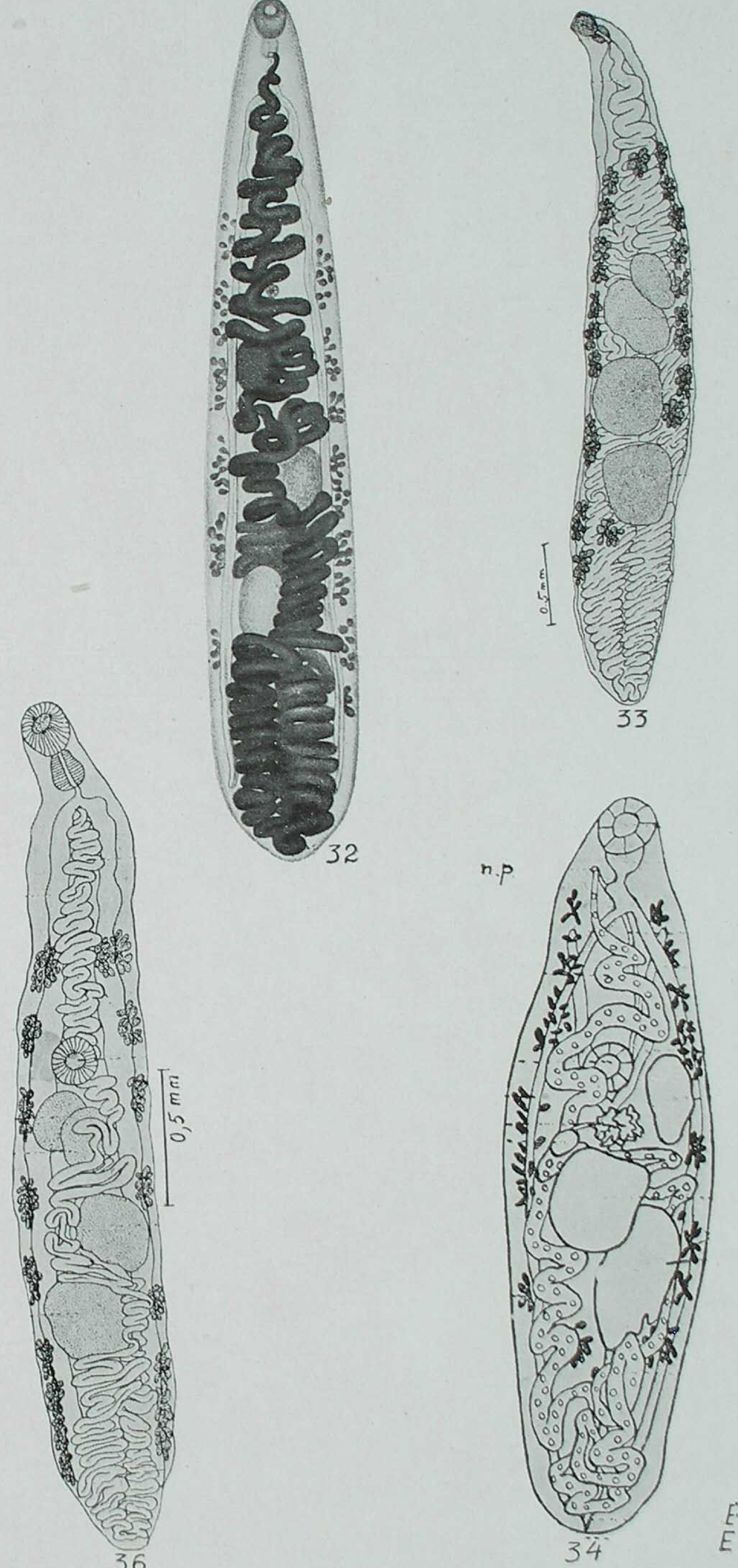

L. Travassos e A. R. Darriba: Trematodeos dos generos Pneumonoeces e Ostiolum. 
Fig. 13a-Pneumonoeces longiplexus-espinhos cuticulares seg. Cort.

Fig. 14-Pneumonoeces longiplexus - segundo Cort.

Fig. 15-Pneumonoeces breviplexus - segundo Stafford.

Fig. 16-Pneumonaeces breviplexus - segundo Cort.

Fig. 17-Pneumonoeces varioplexus -segundo Stafford.

Fig. 18-Pneumonaeces varioplexus (similiplexus)-segundo Stafford.

Fig. 19-Pneumonoeces varioplexus - segundo Cort.

Fig. 20-Pneumonoeces varioplexus - espinhos cuticulares, segundo Cort.

\section{* ESTAMPA LXVIII}

Fig. 21-Pneumonoeces schulzei - original.

Fig. 22-Pneumonoeces campyristis - segundo Klein, 1905.

Fig. 23-Pneumonoeces australis - segundo Johnston.

Fig. 24-Pneumonoeces neivai - segundo Travassos e Artigas.

Fig. 25-Pneumonoeces neivai-photographia; original.

\section{ESTAMPA LXIX}

Fig. 26-Pneumonoeces neivai-photographia do córte de pulmão de $L$. ocellatus, vendo-se um parasito.
Vit. vitellinos; $v$. $s$. vesicula seminal; o. ovario; int. intestino.

\section{ESTAMPA LXX}

Fig. 27-Pneumonoeces neivai-Photographia de córte de pulmão de $L$. ocellatus; $v$. o. ventosa oral; vit. vitellinos; $\boldsymbol{v}$. $s$. vesicula seminal: int. cecos repletos de hematias; $o$. ovario.

Fig. 28-Pneumonoeces neivai-photographia de córte de pulmão de Leplodactylus ocellatus, vendo-se dois parasitos, $v$. o. ventosa oral.

Fig. 29-Pneumonoeces parviplexus - segundo Seely.

\section{ESTAMPA LXXI}

Fig. 30-Pneumonoeces fuelleborni-original.

Fig. 31-Ostiolum medioplexus-espinhos cuticulares-segundo Cort.

Fig. 32-Ostiolum medioplexus - segundo Stafford.

Fig. 33-Ostiolum medioplexus - segundo Cort.

Fig. 34-Ostiolum complexus-segundo Irwin.

Fig. 35-Ostiolum coloradensis-espinhos cuticulares, segundo Cort.

Fig. 36-Ostiolum coloradensis-segundo Cort. 